\title{
Осцилляции де Гааза-ван Альфена в кремниевой наноструктуре в слабых магнитных полях при комнатной температуре
}

\author{
(C) В.В. Романов ${ }^{1}$, В.А. Кожевников ${ }^{1}$, С.T. Tracey $^{1}$, Н.T. Баграев ${ }^{1,2, \text { Ф }}$ \\ ${ }^{1}$ Санкт-Петербургский политехнический университет Петра Великого, \\ 195251 Санкт-Петербург, Россия \\ ${ }^{2}$ Физико-технический институт им. А.Ф. Иоффе Российской академии наук, \\ 194021 Санкт-Петербург, Россия \\ ^ E-mail: Bagraev@mail.ioffe.ru
}

Поступила в Редакцию 19 августа 2019 г.

В окончательной редакции 23 августа 2019 г.

Принята к публикации 23 августа 2019 г.

\begin{abstract}
Полевая зависимость намагниченности кремниевого наносандвича, измеренная при комнатной температуре в слабых магнитных полях, проявляет осцилляции де Гааза-ван Альфена, поведение которых объясняется при условии зависимости эффективной массы носителей от внешнего магнитного поля.
\end{abstract}

Ключевые слова: намагниченность, кремниевый наносандвич, эффективная масса, размерное квантование, эффект де Гааза-ван Альфена.

DOI: 10.21883/FTP.2019.12.48620.9241

\section{1. Введение}

Наблюдение макроскопических квантовых эффектов при комнатной температуре в слабых магнитных полях стало возможным в условиях подавления электронэлектронного взаимодействия, что достигается, например, при наличии оболочек с отрицательной корреляционной энергией, ограничивающих низкоразмерные полупроводниковые структуры [1-3]. Эти эффекты были продемонстрированы при исследовании поведения кинетических коэффициентов (продольная и холловская проводимость, намагниченность) в кремниевом наносандвиче [1], которые проявляют его квантовые свойства вследствие идентификации эффектов Шубникова-де Гааза [2], де Гааза-ван Альфена [3], а также квантовых лестниц продольной проводимости и холловского сопротивления $[1,2]$.

Кроме эффективного подавления электрон-электронного взаимодействия обнаружение вышеперечисленных макроскопических квантовых эффектов обусловлено малым значением эффективной массы дырок в низкоразмерной структуре, в частности в краевых каналах квантовых ям, которое может быть оценено из классического соотношения, связывающего эффективную массу $\left(m_{\mathrm{eff}}\right)$ со скоростью и волновым числом Ферми $\left(v_{\mathrm{F}}, k_{\mathrm{F}}\right)$ :

$$
m_{\mathrm{eff}} v_{\mathrm{F}}=\hbar k_{\mathrm{F}}, \quad k_{\mathrm{F}}=\sqrt{2 \pi p_{2 \mathrm{D}}},
$$

где $p_{2 \mathrm{D}}$ - двумерная плотность дырок в наносандвиче [1].

Скорость носителя на уровне Ферми можно оценить исходя из среднего расстояния между носителями в краевом канале, $\ell$, которое совпадает с линейным размером пиксела, содержащего одиночный носитель в условиях подавления электрон-электронного взаимодействия [1], а также верхнего предела значения диамагнитного тока,
$I_{\text {ind }}$, индуцированного изменяющимся во времени магнитным полем вследствие закона Фарадея [1]. Проведенная таким образом оценка величины эффективной массы в изучаемой наноструктуре дает

$$
m_{\mathrm{eff}} \sim \frac{e \hbar k_{\mathrm{F}}}{\ell I_{\text {ind }}} \sim 10^{-4} m_{e},
$$

где $m_{e}$ - масса свободного электрона, и согласуется по порядку величины с результатом, полученным из измерения эффекта Ааронова-Кашера [2]. Столь малое значение эффективной массы носителей в кремниевом наносандвиче допускает возможность наблюдения макроскопических квантовых эффектов при комнатной температуре в слабых магнитных полях [1-3]. В то же время дальнейшие исследования эффекта де Гаазаван Альфена могут дать ответы на ряд вопросов, касающихся свойств изучаемой структуры, а именно рассматриваемое в настоящей работе влияние эффективной массы носителей на формировании полевой зависимости намагниченности.

В настоящей работе для реализации этой цели были выполнены измерения намагниченности кремниевого наносандвича, где проявляется эффект де Гааза-ван Альфена при комнатной температуре, в режиме дискретной развертки магнитного поля с шагом $5 Э$ в интервале напряженностей от 100 до 1100 Э, и проведен ее анализ. Выбранный нами метод измерения намагниченности допускает возможность использования различных подходов для описания и интерпретации эксперимента, в том числе формализма равновесной термодинамики, что обеспечено реализацией квазистатического процесса в ходе измерений намагниченности.

\section{2. Эксперимент}

Измерения намагниченности методом Фарадея выполнялись на установке Faraday Balance, созданной на базе 


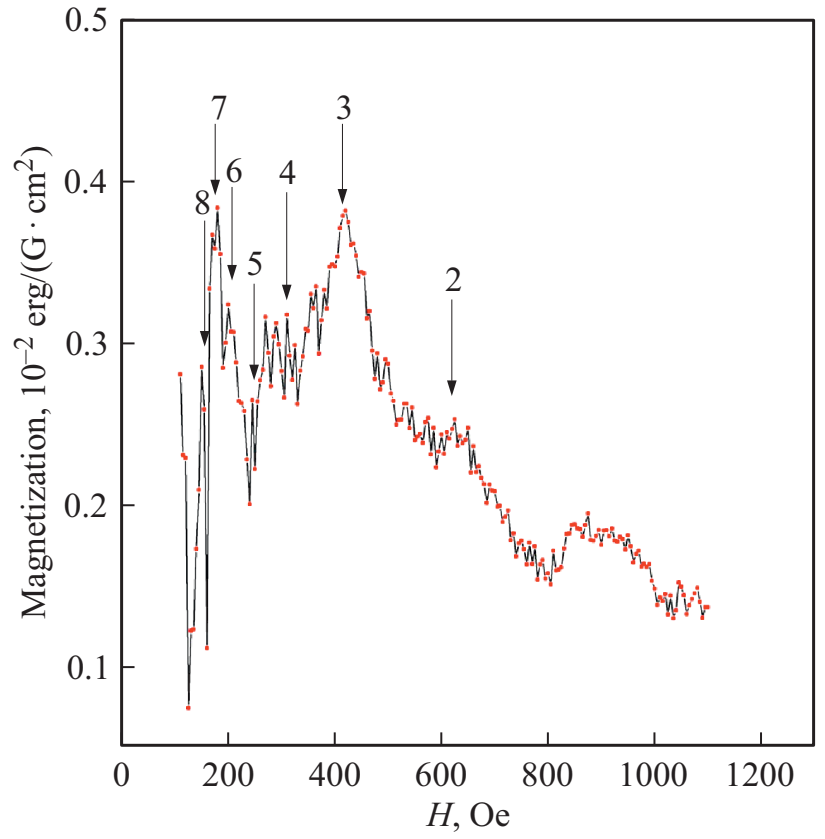

Рис. 1. Полевая зависимость с осцилляциями де Гаазаван Альфена для кремниевого наносандвича при комнатной температуре. Указаны значения фактора заполнения.

спектрометра MGD 312 FG, при комнатной температуре в магнитных полях напряженностью до 1100 Э. Образцы помещались во внешнее магнитное поле в кварцевой чашке, подвешенной на тонкой кварцевой нити. Ориентация образца во внешнем магнитном поле определялась с учетом рекомендаций, высказанных в [4] касательно особенностей используемого нами метода измерений. Это позволило избежать усреднения магнитного поля на линейном размере структуры, которое могло привести к тушению осцилляций. Развертка магнитного поля осуществлялась в пошаговом режиме с заданным значением шага $\Delta H$, причем регистрация интенсивности взаимодействия с магнитным полем осуществлялась при достижении образцом состояния термодинамического равновесия, что контролировалось автоматизированной системой управления измерениями с помощью специально разработанного программного обеспечения. Калибровка установки проводилась с помощью эталонного образца, в качестве которого использовался монокристалл магнитно-чистого фосфида индия с восприимчивостью $\chi=-313 \cdot 10^{-9} \mathrm{~cm}^{3} /$ г. Масса образца определялась на весах ВР $211 \mathrm{D}$ с точностью до $10^{-5}$ г. Кривая намагниченности образца, полученная при изменении внешнего магнитного поля с шагом $\Delta H=5$ Э, приведена на рис. 1 .

Особо отметим, что образец перед началом измерений не подвергался каким-либо внешним воздействиям, таким как оптическое облучение, предварительная выдержка во внешних электрическом или магнитном полях, термическая обработка, способным на время измерений вывести его из равновесного состояния.
Таким образом, полученная в эксперименте полевая зависимость представляет собой совокупность дискретных значений намагниченности, отображающих поведение системы в квазистатическом процессе, т.е. при ее прохождении через ряд термодинамически равновесных состояний, причем регистрируемый отклик системы каждый раз отображал ее равновесное состояние во внешнем магнитном поле.

Все сказанное выше дает нам основания использовать формализм равновесной термодинамики для интерпретации полученных результатов.

\section{3. Результаты эксперимента}

Зависимость на рис. 1 содержит хорошо разрешенные осцилляции де Гааза-ван Альфена до значений фактора заполнения $v=8$ включительно. При этом с ростом внешнего магнитного поля (уменьшением фактора заполнения) наблюдается тенденция к уменьшению амплитуды осцилляций намагниченности.

\section{4. Обсуждение}

Полученные при комнатной температуре осцилляции де Гааза-ван Альфена интересны прежде всего с позиций анализа их амплитуды как функции фактора заполнения $v$. При этом следует учитывать, что для количественного анализа полученных экспериментальных данных расчеты намагниченности двумерных электронных структур должны основываться на предварительно выбранной модели плотности состояний (DOS). Поэтому наиболее критичным выбор модели, безусловно, является при проведении низкотемпературных экспериментов. Как было показано в ряде работ [5-8], адекватное описание экспериментальные кривые намагниченности низкоразмерных структур получают при использовании DOS с небольшим гауссовым уширением уровней Ландау и энергетически независимой основой.

Обращаясь к зависимости амплитуды осцилляций намагниченности от фактора заполнения в различных исследованных структурах, отметим, что для идеализированной системы (температура $T=0 \mathrm{~K}$ ) характерны осцилляции с неизменным размахом при всех значениях фактора заполнения [5]. Однако анализ результатов вычислений намагниченности как функции фактора заполнения при конечных температурах специфически для двумерных образцов выявляет монотонный рост амплитуды осцилляций с ростом внешнего магнитного поля [5-7]. Этот вывод согласуется с опубликованными к настоящему времени результатами экспериментальных исследований эффекта де Гааза-ван Альфена в двумерных электронных системах, проведенных при низких температурах, от 0.3 до $30 \mathrm{~K}$, в сильных магнитных полях $(\sim 10$ Тл), и коррелируют с измерениями удельной теплоемкости двумерных электронов [8]. Отметим, что эксперименты в основном проводились на модулированно-легированном гетеропереходе 
$\mathrm{AlGaAs} / \mathrm{GaAs}$ со значением эффективной массы носителя $\sim 10^{-2} m_{e}[5-9]$, причем эффект де Гааза-ван Альфена наблюдался после предварительного оптического воздействия на образец $[5-7,9]$.

Обратимся теперь к полевой зависимости намагниченности, полученной при наблюдении эффекта де Гаазаван Альфена в кремниевом наносандвиче в слабых магнитных полях при комнатной температуре (рис. 1). Очевидно, что экспериментальная зависимость, представленная на рис. 1, свидетельствует об уменьшении размаха осцилляций с ростом напряженности внешнего магнитного поля. Кроме того, из рис. 1 следует, что намагниченность исследуемого образца характеризуется сложной зависимостью от приложенного магнитного поля, представляющей собой быстро осциллирующую намагниченность, обусловленную квантованием Ландау, относительно сравнительно медленно меняющейся с магнитным полем намагниченности квантовой ямы, ограниченной краевыми каналами. Анализируя возможные факторы, влияющие на формирование экспериментальной зависимости, а также результаты измерений продольной проводимости исследуемой структуры во внешнем магнитном поле [1], можно предположить, что эффективная масса носителей в кремниевом наносандвиче зависит от величины приложенного магнитного поля.

Для качественного описания поведения амплитуды осцилляций в системе с эффективной массой, зависящей от напряженности внешнего магнитного поля, мы снова можем обратиться к идеализированной системе. Полученная из термодинамических соотношений зависимость энергии системы уровней Ландау от внешнего поля в этом случае принимает вид, представленный на рис. 2.

Кривая намагниченности, вычисленная дифференцированием энергетического спектра заполненных уровней

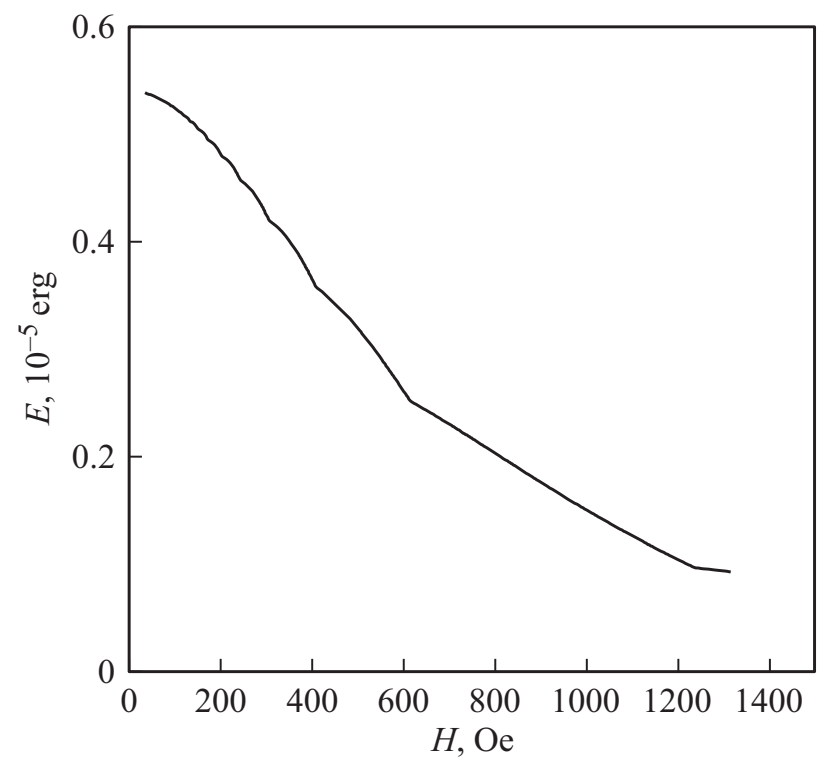

Рис. 2. Зависимость энергии $E$ системы уровней Ландау от внешнего поля $H$ для изкоразмерной системы с эффективной массой, возрастающей с увеличением напряженности магнитного поля.

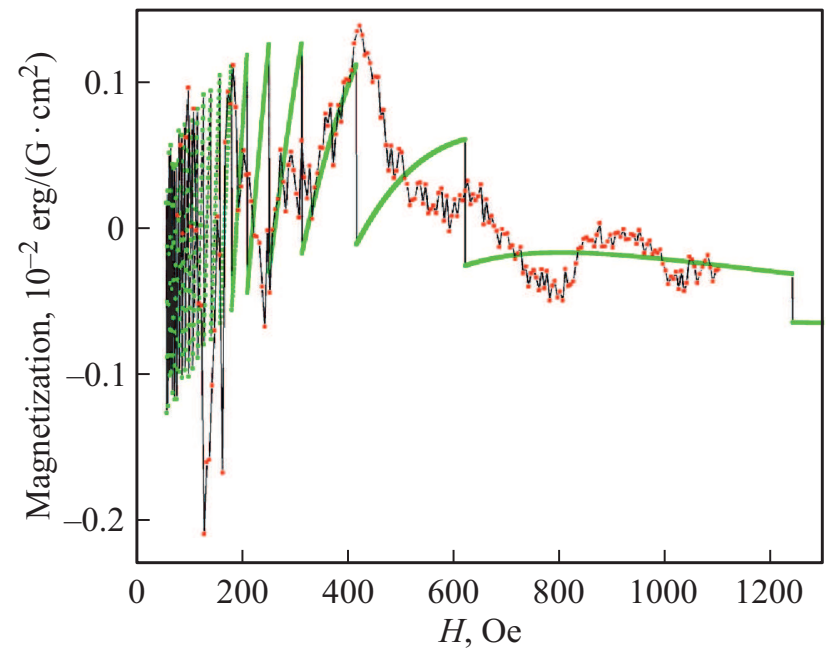

Рис. 3. Суперпозиция экспериментальной кривой и „идеализированной“ полевой зависимости намагниченности кремниевого наносандвича, вычисленной в приближении переменной эффективной массы в рамках описания осцилляций де Гаазаван Альфена.

Ландау по напряженности внешнего магнитного поля при постоянной температуре, для системы с переменной массой носителя показывает убывание амплитуды осцилляций с уменьшением фактора заполнения и выявляет немонотонное поведение медленно меняющейся намагниченности (рис. 3). Таким образом, как следует из сопоставления с экспериментальной кривой, использование приближения зависящей от магнитного поля эффективной массы носителей позволяет адекватно описать эксперимент (см. рис. 3).

Следует отметить, что наблюдаемая в эксперименте зависимость эффективной массы от напряженности внешнего магнитного поля может быть представлена аналитически. Наилучшее согласие с экспериментом достигается, если принять, что эффективная масса носителя изменяется с полем по закону

$$
m_{\mathrm{eff}}=\left(1+\alpha H^{2}\right) \cdot 6 \cdot 10^{-5} m_{e},
$$

где $m_{\mathrm{eff}}-$ эффективная масса носителя; $\alpha=3 \cdot 10^{-6} \ni^{-2}-$ параметр, $H-$ напряженность внешнего магнитного поля, $m_{e}-$ масса свободного электрона.

Согласно (2), в диапазоне полей от 100 до 1000 Э эффективная масса носителя монотонно изменяется от $6 \cdot 10^{-5}$ до $2.4 \cdot 10^{-4}$ массы свободного электрона.

\section{5. Заключение}

Если исходить из сопоставления экспериментальной и расчетной зависимостей (рис. 3), феноменология высокотемпературного эффекта де Гааза-ван Альфена допускает истолкование в модели переменной массы носителей, причем с увеличением внешнего магнитного поля $m_{\mathrm{eff}}$ возрастает согласно (2). 
Полевая зависимость намагниченности кремниевого наносандвича, измеренная при комнатной температуре (рис. 1), имеет ряд особенностей, существенно отличающих ее от зависимостей, полученных в исследованиях эффекта де Гааза-ван Альфена в низкоразмерных структурах при низких температурах в сильных магнитных полях [5-7,9]. Анализ наблюдаемых особенностей позволяет сделать важные заключения об основных свойствах исследуемой низкоразмерной структуры. В настоящей работе показано, что носители заряда в наносандвиче характеризуются низким значением эффективной массы, причем наблюдаемый эффект де Гааза-ван Альфена получает адекватное описание в приближении эффективной массы носителя, зависящей от внешнего магнитного поля.

В рамках обсуждаемой модели осцилляций де Гаазаван Альфена следует учесть, что, как отмечено выше, электрон-электронное взаимодействие между носителями (дырками) в краевых каналах, ограничивающих сверхузкую кремниевую квантовую яму $p$-типа проводимости, в значительной степени подавлено вследствие присутствия дипольных центров бора с отрицательной корреляционной энергией, что, по-видимому, приводит к уменьшению эффективной массы одиночных дырок в краевых каналах. Однако при увеличении внешнего магнитного поля кванты магнитного потока захватываются в пикселы, содержащие одиночные дырки внутри краевого канала, вследствие чего эффективная масса последних начинает возрастать. Таким образом, увеличение числа квантов магнитного потока в фазовом пространстве носителя может приводить к эффективному „утяжелению“ носителей в краевом канале, что, по-видимому, определяет наблюдаемую в эксперименте зависимость амплитуды осцилляций де Гааза — ван Альфена от магнитного поля. Безусловно, наблюдаемое явление и предложенный для его объяснения механизм предполагают дальнейшее исследование и обсуждение.

\section{Конфликт интересов}

Авторы заявляют об отсутствии у них конфликта интересов.

\section{Список литературы}

[1] N.T. Bagraev, V.Yu. Grigoryev, L.E. Klyachkin, A.M. Malyarenko, V.A. Mashkov, V.V. Romanov. Semiconductors, 50 (8), 1025 (2016).

[2] N.T. Bagraev, L.E. Klyachkin, V.V. Romanov, A.I. Ryskin. Low Temp. Phys., 40 (4), 352 (2014).

[3] N.T. Bagraev, V.Yu. Grigoryev, L.E. Klyachkin, A.M. Malyarenko, V.A. Mashkov, V.V. Romanov, N.I. Rul'. Low Temp. Phys., 43 (1), 1 (2017).

[4] D. Shoenberg. Magnetic oscillations in metals (Cambridge, University Press, Cambridge, 1984).

[5] M.P. Schwarz, M.A. Wilde, S. Groth, D. Grundler, Ch. Heyn, D. Heitmann. Phys. Rev. B, 65, 245315 (2002).

[6] I.D. Vagner, Tsofar Maniv, E. Ehrenfreund. Phys. Rev. Lett., 51, 1700 (1983).
[7] J.G.E. Harris, R. Knobel, K.D. Maranowski, A.C. Gossard, N. Samarth, D.D. Awschalom. Phys. Rev. Lett., 86, 4644 (2001).

[8] E. Gornik, R. Lassnig, G. Strasser, H.L. Stormer, A.C. Gossard, W. Wiegmann. Phys. Rev. Lett., 54, 1820 (1985).

[9] J.P. Eisenstein, H.L. Stormer, V. Narayanamurti, A.Y. Cho, A.C. Gossard, C.W. Tu. Phys. Rev. Lett., 55, 875 (1985).

Редактор Л.В. Шаронова

\section{de Haas-van Alphen oscillations observed in silicon nanosandwich at room temperature in weak magnetic fields}

\author{
V.V. Romanov ${ }^{1}$, V.A. Kozhevnikov ${ }^{1}$, C.T. Tracey ${ }^{1}$, \\ N.T. Bagraev ${ }^{1,2}$
}

${ }^{1}$ Peter the Great St. Petersburg Polytechnic University, 195251 St. Petersburg, Russia

2 loffe Institute,

194021 St. Petersburg, Russia

Abstract The field dependence of magnetization of silicon nanosandwich measured at the room temperature in weak magnetic fields demonstrates de Haas-van Alphen oscillations whose behavior is interpreted within frameworks of effective mass of carriers dependent on external magnetic field. 